\title{
2826. Structural damage detection based on cloud model and Dempster-Shafer evidence theory
}

\author{
Liang Tian', Huiyong Guo ${ }^{2}$, Xinyu Zhou ${ }^{3}$, Yushan Wang ${ }^{4}$ \\ ${ }_{1,2,3}$ Key Laboratory of New Technology for Construction of Cities in Mountain Area, \\ Ministry of Education, Chongqing, 400045, China \\ ${ }^{1,2,3}$ School of Civil Engineering, Chongqing University, Chongqing, 400045, P. R. China \\ ${ }^{4}$ School of Water Conservancy and Architectural Engineering, Shihezi University, Shihezi, 832003, China \\ ${ }^{2}$ Corresponding author

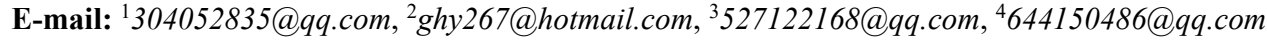

Received 20 March 2017; received in revised form 1 August 2017; accepted 10 August 2017

DOI https://doi.org/10.21595/jve.2017.18361

Check for updates

\begin{abstract}
Cloud model and D-S theory have been widely used in uncertainty reasoning. Meanwhile, modal strain energy and Inner Product Vector are also utilized as damage-sensitive features to detect structural damage. In this paper, a new structural damage identification approach is proposed based on Dempster-Shafer theory and cloud model. Cloud models were created to make uncertainty reasoning in damage structures by modal strain energy and the Inner Product Vector of acceleration. Then the results of the two methods were combined by using the Dempster-Shafer theory. Due to the classical D-S theory involves counter - intuitive behavious when the high conflicting evidences exists, the distance function was introduced to correct the conflict factor $K$ and combine the evidences. Moreover, a model of simple beam was created to verify the feasibility and accuracy for the single-damage and the multiple-damage. The effects of noise on damage detection were investigated simultaneously. The results show that the method has strong anti-noise ability and high accuracy.
\end{abstract}

Keywords: damage identification, cloud model, D-S theory, mode strain energy, inner product vector.

\section{Introduction}

In the past two decades, the security of building structure has received increasingly concern both at home and abroad especially the damage detection for structure itself. As a result of the structural damage, the local rigidity of the structure will descend while the modal parameters change. Therefore, most of researchers make the damage detection analysis based on modal parameters and the structural dynamic responses. Koh [1] has made damage detection in Suspension Bridge based on the modal correlation, and Shi Z. Y. [2] presented a method for structural damage localization and damage degree analysis by the incomplete modal displacement change before and after structure damaged. Fan [3] has made damage research on plate structure, using the wavelet transform of structural vibration mode and modal shape, obtained good results. S. M. Seyedpoor [4] has put forward a method which is divided into two stage to detect structural damage, based on the modal strain energy calculated by the particle swarm optimization. Guo [5] has proposed a method based on evidence theory and modal strain energy to make damage analysis. And ShaoFei Jiang [6] combined the Artificial Neural Network with the Dempster-Shafer theory [7, 8], and its effectiveness has also been proved in the research.

In the prior context, we find that the modal strain energy has been widely applied in structural damage detection. On the other hand, there are also some damage research based on the Inner Product Vector (IPV). The IPV is related to the mode shapes and can be directly calculated by the time domain vibration responses. Zhichun Yang [9] has proposed a method based on the changes in IPV, and damage detection experiments of shear frame structure are presented to illustrate the reliability and effectiveness of the method. Muyu Zhang [10] has formulated a new approach to detect the damage based on the auto correlation function, and the auto correlation function of the vibration response signals from different measurement points can be used to detect damage 
accurately even when the damage is very small. But most of above methods have difficulties with uncertainty in structural damage identification area.

In this paper, a new method is proposed to detect the structural damage based on cloud model and Dempster-Shafer theory. D-S theory and cloud model are both effective ways to deal with uncertainty, and have been widely used in mechanical field, electronic field and medical field. In the field of structural damage identification, Qingguo Fei [11] used the modal strain energy change ratio by model shapes of different orders to locate the damage element of a fix-end beam, then applied the D-S theory to fuse the results of different orders. Hui Li [12] proposed a damage identification method based on the combination of ratification neural network, D-S evidence theory and the Shannon entropy and used a three-dimensional finite element model of the Binzhou Yellow River Highway Bridge to verify the accuracy of the method. Qifeng Zhou [13] has proposed an intelligent detection method which combined the posteriori probability support vector machines and D-S theory to detect the location and extent of structural damage. Since it is difficult to solve uncertain problem in structural damage identification only by D-S theory, cloud model is introduced to combine with D-S theory. At present, the cloud model is seldom used in the field of structural damage identification. In this paper, firstly the cloud model is introduced, which was proposed by Deyi Li in 1995 [14]. Cloud model is an effective tool to deal with the uncertainty and can realize the conversion between qualitative concepts and quantitative values. Then the Demspster-Shafer theory is introduced, which is originated from Dempster's work on multi-valued mapping in the 1960s, and has been widely used recently in the fields of uncertain reasoning. Then the modal strain energy and the acceleration IPV are used to formulate cloud models separately. Next combine the two results by modal energy and IPV with the improved Demspster-Shafer theory.

Theoretically the method has strong anti-noise ability and high accuracy. Firstly, multiply cloud reasoning are performed in both two cloud generators, which ensure the accuracy of the reasoning result and improve the anti-noise ability. Secondly, D-S theory is improved to eliminate the possibility of wrong combined results. Meanwhile, the identification results of two cloud generators are combined according to the combination rules. Damage detect of a simple beam is used to verify the method. The results show that the new method has good anti-noise ability, effectiveness for damage. And as a supplement, the method based on modal strain energy dissipation rate is adopted to compare with the new method in based on cloud model and D-S theory. The results show that the new method has stronger anti-noise ability and higher accuracy than modal strain energy dissipation rate.

\section{Cloud model}

In cloud model, three numerical characteristics are used to describe the information uncertainty, Expectation Ex, Entropy En, and Excess Entropy He [15-17]. The detailed descriptions are listed in the following context.

Ex: A point that can stand the qualitative concept to the maximal degree in the whole universe space. It is also the position of the center of gravity of the cloud.

$E n$ : The discrete degree of cloud droplet that represents the qualitative concept can be reflected by this numerical characteristic, and it shows the connection between the randomness and fuzziness.

$H e$ : The measurement of the entropy. In another word, it is the entropy of the entropy. This numerical characteristic reflects the uncertainty aggregation extent of all the points that contribute to the qualitative concept $C$ in the quantitative universe $U$ and the magnitude of the randomness of the certainty degree $u(x)$.

In a word, the three numerical characteristic combine the fuzziness with the randomness perfectly, and shows the mutual mapping between the qualitative concept and the quantitative numeric. In this paper, we use the three numerical characteristic to construct a normal cloud generator to realize the transformation between the qualitative concept and the quantitative 
numeric.

The cloud generators can be divided into normal cloud generator and backward cloud generator. Both the position coordinates of a cloud droplet in the quantitative universe space and the qualitative extent can be obtained by inputting the three numerical characteristic $(E x, E n, H e)$ and the quantity of cloud droplets that need to be generated in the normal cloud generator. Similarly, the backward cloud generator is the inverse process of the normal cloud generator.

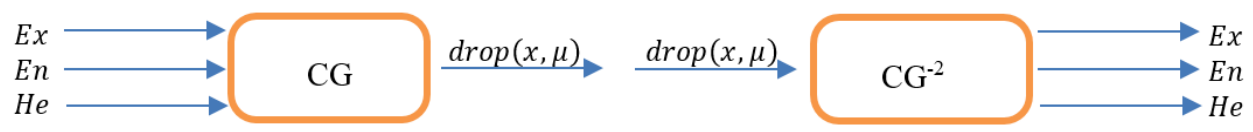

Fig. 1. Normal cloud generator and backward cloud generator

The normal cloud generator algorithm [18]:

a) Generating a normal random number $X r$ according to the three numerical characteristic ( $E x$, $E n, H e$ ), and the expectation of $X r$ is $E x$ and the standard deviation is $E n$.

b) Generating a normal random number $E n$, of which the expectation is $E n$ and the standard deviation is $\mathrm{He}$.

c) Generating the cloud droplet $\left(x_{r}, \mu_{r}\right)$ by $\mu_{r}=\exp \left[-\left(x_{r}-E x\right)^{2} /\left(2 E n^{\prime 2}\right)\right]$.

d) Repeat the above procedure until $N$ cloud droplets were generated.

The backward cloud generator algorithm [19]:

a) $\bar{X}=\frac{1}{N} \sum_{r=1}^{N} x_{r}, M_{1}^{*}=\frac{1}{N} \sum_{r=1}^{N}\left|x_{r}-\bar{X}\right|, S^{2}=\frac{1}{N-1} \sum_{r=1}^{N}\left(x_{r}-\bar{X}\right)^{2}$.

b) $\widehat{E x}=\bar{X}$.

c) $\widehat{E n}=\sqrt{\frac{\pi}{2}} \times \frac{1}{N} \sum_{r=1}^{N}\left|x_{r}-\widehat{E x}\right|$.

d) $\widehat{H e}=\sqrt{\left|S^{2}-\widehat{E n}^{2}\right|}$.

Given three digital characteristics $E x, E n, H e$ and a particular number $x_{0}$, the generator could produce the cloud droplet $\left(x_{0}, \mu\right)$. This kind of generators is called forward cloud generator as well as $X$ conditions cloud generators. By contrast, given the three digital characteristics and a particular $Y=u_{i}$, the generator could produce cloud droplet $\left(x_{i}, u_{i}\right)$. This kind of generators is called backward cloud generator as well as $Y$ conditions cloud generators.

A qualitative rule could be described as if $A$ then $B$, only single condition and single rule generators are discussed in this paper. The algorithm is listed as follows:

Input: the digital characteristics of forward cloud qualitative concept $\mathrm{C} 1\left(E x_{a}, E n_{a}, H e_{a}\right)$ and backward cloud qualitative concept $\mathrm{C} 2\left(E x_{b}, E n_{b}, H e_{b}\right)$, the quantitative number $x_{a}$ for $\mathrm{C} 1$.

Output: the quantitative number $x_{b}$ got form certainty $u$ for $\mathrm{C} 2$.

Detailed procedures [20]:

1) Generate a normal random number $E n_{a}^{\prime}$, of which expectation is $E n_{a}$, and standard deviation is $\mathrm{He}_{a}$.

2) Calculate the certainty by $\mu=\exp \left[-\left(x_{a}-E x_{a}\right)^{2} /\left(2 E n_{a}^{\prime 2}\right)\right]$.

3) Generate a normal random number $E n_{b}^{\prime}$, of which expectation is $E n_{b}$, and standard deviation is $H e_{b}$.

4) If $x_{a}>E x_{a}, x_{b}=E x_{b}+E n_{b}^{\prime} \sqrt{-2 \ln \mu}$.

5) If $x_{a} \leq E x_{a}, x_{b}=E x_{b}-E n_{b}^{\prime} \sqrt{-2 \ln \mu}$.

From the above texts, we can know that the degree of uncertainty could be passed on by cloud generators. Firstly, a quantitative value $x_{a}$ was input into the forward cloud generator, so we can get the certainty $\mu$ by the cloud generator. Then the certainty $\mu$ was input into the backward cloud generator and a quantitative value $x_{b}$ was obtained. 


\section{Modal strain energy index and inner product vector of acceleration}

Structural damage will change the mass, stiffness and damping properties of a structure, thus leading to the change of structural frequency and vibration mode. Therefore, various methods have been introduced to identify the damage in structure based on comparing the response data of the structure before and after damage. One of the most popular methods is that one based on using the strain energy of a structure.

Assuming that the structure is divided into $n$ elements, the modal strain energy of $j$ th structural element for $i$ th mode before and after damage can be expressed by a matrix notation as:

$$
\begin{aligned}
M S E_{i j} & =\frac{1}{2}\left\{\varphi_{i}\right\}^{T}\left[K_{j}\right]\left\{\varphi_{i}\right\}, \\
M S E_{i j}^{d} & =\frac{1}{2}\left\{\varphi_{i}^{d}\right\}^{T}\left[K_{j}\right]\left\{\varphi_{i}^{d}\right\},
\end{aligned}
$$

where $\varphi_{i}$ is the $i$ th-order modal vibration mode of the structure, $\varphi_{i}^{d}$ is the $i$ th-order modal vibration mode of the damaged structure, $K_{j}$ is the stiffness matrix of the $j$ th element.

Hui Liu [21] introduced a method to identify the structural damage by modal strain energy dissipation rate. The damage coefficient of $f$ th element can be expressed as:

$c_{f}=\frac{\left|M S E_{f}^{d}-M S E_{f}\right|}{\left|M S E_{f}^{d}-M S E_{f}\right|+M S E_{f}}$.

In recent years, a new approach to detecting structure damage by using the inner product vector of the measured vibration responses was proposed. This kind of method has the advantage of high accuracy in damage detection. In this paper, the inner product is based on the cross correlation functions of displacement response under white noise excitation. Assuming that there exists a series of response measurement points in the structure, and the acceleration inner product vectors can be calculated by the inner product of the time domain vibration responses of each point under white noise excitation when the time lag equals to zero. The acceleration inner product vector $R_{i p v}$ is defined as [22]:

$\widehat{R} I P V=\frac{1}{N}\left[\left\langle x_{i_{1}}, x_{j}\right\rangle,\left\langle x_{i_{2}}, x_{j}\right\rangle, \ldots,\left\langle x_{i_{p}}, x_{j}\right\rangle\right]^{T}$,

where $N$ : the numbers of the response measurement points; $X_{i p}$ : the time series of structure excitation response of measurement points; $X_{j}$ : the time series of structure excitation response of reference point.

In this paper, the acceleration inner product vector $R_{i p v}$ is normalized by its maximum value:

$R_{i p v}=\frac{\hat{R}_{i p v}}{\max \left(\hat{R}_{i p v}\right)}$.

The structural modal parameter can be changed when the structure is damaged, as well as abrupt changes in mode shapes. Therefore, the inner product vector of the damaged structure may exhibit abrupt changes. So, the damage index can be formulated as:

$D_{i p v}=R_{i p v, i}^{d}-R_{i p v, i}^{u}$,

where $R_{i p v, i}^{d}$ and $R_{i p v, i}^{u}$ indicate the ith element in the inner product vectors of the intact and 
damage structures, respectively. Sometimes, the change of the inner product vector is not obvious in tiny structure damages, so a new damage index is formulated to make the damage location clear as:

$D^{\prime}{ }_{i p v, i}=D_{i p v, i+1}-2 D_{i p v, i}+D_{i p v, i-1}$.

\section{D-S evidence theory}

D-S evidence theory is originated from Dempster's work on multi-valued mapping in the 1960 s, and has been widely used recently in the fields of uncertain reasoning.

Let $m_{1}, m_{2}$ be two pieces of evidence in the same frame of discernment $\Theta$, and the evidence fusion rule is listed as follows [23]:

$m(A)= \begin{cases}0, & A=\emptyset, \\ K^{-1} \sum_{A_{i} \cap B_{j}=A} m_{1}\left(A_{i}\right) m_{2}\left(B_{j}\right), & A \neq \emptyset,\end{cases}$

where $A, A_{i}, B_{j} \in 2^{\Theta}, K=1-\sum_{A_{i} \cap B_{j}=\emptyset} m_{1}\left(A_{i}\right) m_{2}\left(B_{j}\right)$, which measure the conflict degree between the two pieces of evidence. In Eq. (8), the two pieces of evidence completely conflict when $K=0$, and in this situation the fusion rule is unavailable. If $K \neq 0$, the $m(A)$ satisfies $\sum m(A)=1$. When $K \rightarrow 0$, the two pieces of evidence are in high conflict, and an illogical result is the traditional D-S fusion rule. To improve the fusion accuracy of the D-S theory, distance function defined by Jousselme [24] is introduced. The distance function is defined as follows:

$d\left(m_{1}, m_{2}\right)=\sqrt{\frac{1}{2}\left(m_{1}-m_{2}\right)^{T} D\left(m_{1}-m_{2}\right)}$,

where $D=\left(D_{i j}\right)$ is a matrix with order $2^{N} \times 2^{N}$. And the specific calculation formula of Eq. (9) is given by:

$d\left(m_{1}, m_{2}\right)=\sqrt{\frac{1}{2}\left(\left\langle m_{1}, m_{1}\right\rangle+\left\langle m_{2}, m_{2}\right\rangle-2\left\langle m_{1}, m_{2}\right\rangle\right)}$,

where $\left\langle m_{1}, m_{2}\right\rangle$ is the inner product of the two vectors. Combined with the traditional conflict factor $K$ and the distance function $d\left(m_{1}, m_{2}\right)$, the new conflict factor between evidence is defined as follows:

$K_{r m s}=\sqrt{\frac{1}{2}\left[K^{2}+d\left(m_{1}, m_{2}\right)^{2}\right]}$.

\section{Numerical example of cloud reasoning}

To apply the above method to damage identification of structure, a simply supported beam model is used as Fig. 2. There are 20 elements of the simply beam and its overall length is $6 \mathrm{~m}$, cross section area is $0.005 \mathrm{~m}^{2}$, moment of inertia is $1.67 \mathrm{~m}^{4}$, elastic modulus is $32 \mathrm{GPa}$, density is $2500 \mathrm{~kg} / \mathrm{m}^{3}$.

From the above information, we know the model has 39 degrees of freedom. And the paper used the Matlab software to establish finite element model, ignore the model error. Two different damage scenarios given in Table 1 are considered in the model to test the proposed method. 


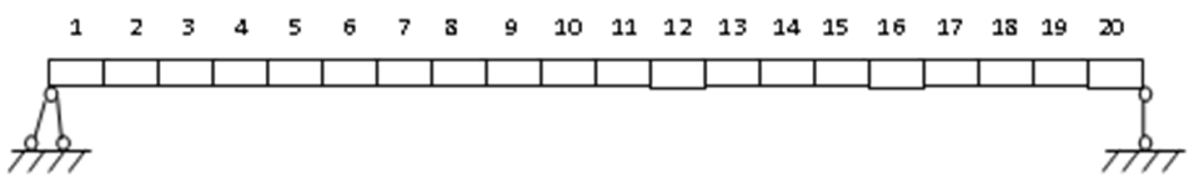

Fig. 2. Euler-Bernoulli beam

Table 1. Two different damage scenarios induced in model

\begin{tabular}{|c|c|c|c|}
\hline \multicolumn{2}{|c|}{ Scenario 1 } & \multicolumn{2}{c|}{ Scenario 2 } \\
\hline Element number & Damage ratio & Element number & Damage ratio \\
\hline 8 & 0.2 & 6 & 0.2 \\
\hline 14 & 0.2 & 15 & 0.2 \\
\hline
\end{tabular}

Here we formulate the mapping $f_{1}$ to connect the element stiffness damage degree $C_{f}$ and the modal strain energy after structure damaged, as well as the mapping $f_{2}$ to connect the $C_{f}$ and the acceleration inner product vector after structure damaged. Forward cloud generator A1 is built by the damaged modal strain energy and A2 is built by the damaged acceleration inner product vector of every element. Meanwhile, the backward cloud generator B1 and B2 are built by the damage degree $C_{f}$ of every element, respectively. Then the rule base of cloud reasoning is formulated.

Suppose that the backward generator is composed of five qualitative concepts: Damage Level 1-Level 5. Each digital characteristic of qualitative concept calculated by the gray cloud model is listed in Table 2.

Table 2. Digital characteristics of qualitative concept in variable degrees of damage

\begin{tabular}{|c|c|c|c|c|c|}
\hline & Level 1 & Level 2 & Level 3 & Level 4 & Level 5 \\
\hline Boundaries & $(0,0.1)$ & $(0.1,0.2)$ & $(0.2,0.3)$ & $(0.3,0.4)$ & $(0.4,0.5)$ \\
\hline$E x$ & 0.05 & 0.15 & 0.25 & 0.35 & 0.45 \\
\hline$E n$ & 0.0167 & 0.0167 & 0.0167 & 0.0167 & 0.0167 \\
\hline$H e$ & 0.0017 & 0.0017 & 0.0017 & 0.0017 & 0.0017 \\
\hline
\end{tabular}

Then, the backward cloud generator B1 can be obtained by the forward cloud generator A1 in Matlab software.

Table 3 is the canonical matrix formulated by expectation of damaged MSE in variable degrees of damage. Entropy of damaged MSE is also necessary to formulate the cloud model, which is shown in Table 4. And the excess entropy of damaged MSE is one tenth of entropy according to the grey cloud model. The specific calculation steps are listed as follows:

1) Suppose there are $m$ evaluation indexes and $n$ evaluation objects to formulate the canonical matrix $A=\left(a_{r f}\right)_{n \times m}$.

2) Calculate the entropy of the $f$ th index:

$S_{f}=1+\frac{\left(\sum_{r=1}^{n} x_{r f} \ln x_{r f}\right)}{\ln (n)}$

$x_{r f}=\frac{a_{r f}}{\sum_{r=1}^{n} a_{r f}}$.

3) Calculate the soften factor of the $f$ th index:

$w_{f}=\frac{S_{f}}{\sum_{f=1}^{m} S_{f}}, \quad 0 \leq w_{f} \leq 1, \quad \sum_{f=1}^{m} w_{f}=1$.

4) Give risen order to each evaluation index in matrix A and denoted by $a_{r f}^{\prime}$, by which the threshold value of the $f$ th evaluation index to the $r$ th gray class can be obtained, as well as the 
standard value interval:

$\theta_{r f}=\frac{1}{2}\left(a_{r f}^{\prime}+a_{r(f+1)}^{\prime}\right)\left(1+w_{f}\right), \quad f=1,2, \ldots, m-1$,

$\theta_{r f}=1.5 \times a_{r f}^{\prime}, \quad f=m$,

$\theta_{r f}, L=0.95 \times a_{r f}^{\prime}, \quad f=1,2, \ldots, m$,

$\theta_{r f}, R=\theta_{r f}, \quad f=1,2, \ldots, m$,

where $\theta_{r f}, L$ : the left endpoint of the standard value interval, $\theta_{r f}, R$ : the right endpoint of the standard value interval.

5) Apply the canonical matrix of expectation of damaged MSE in variable degrees of damage into gray model.

Peak value $C_{s}$ : the expectation of damaged MSE in variable degrees of damage.

Threshold value $R_{s}$ and $L_{s}$ : the endpoints of the standard value interval $\theta_{r f}, L$ and $\theta_{r f}, R$.

6) $E n=R_{s}-L_{s} / 6, H e=k=E n / 10$.

Table 3. Expectation of damaged MSE in variable degrees of damage

\begin{tabular}{|c|c|c|c|c|c|}
\hline Element & Level 1 & Level 2 & Level 3 & Level 4 & Level 5 \\
\hline 1 & 2841378885 & 3158871445 & 3530452308 & 3969275783 & 4493638057 \\
\hline 2 & 563314131.9 & 661809738.9 & 789239381.9 & 958654589.8 & 1191963547 \\
\hline 3 & 237085075.8 & 288903906.8 & 359955502.4 & 461228971.6 & 613024267 \\
\hline 4 & 1054678417 & 1278960849 & 1582467110 & 2006951696 & 2625347900 \\
\hline 5 & 1787482817 & 2139114524 & 2604416327 & 3237498089 & 4128365920 \\
\hline 6 & 1696276664 & 2019134078 & 2443316748 & 3015882137 & 3814571320 \\
\hline 7 & 979398889 & 1174941312 & 1435326045 & 1792675840 & 2301751997 \\
\hline 8 & 428936988.8 & 518133305.4 & 638443741.9 & 806313141.2 & 1050811901 \\
\hline 9 & 635392118.8 & 770379695.4 & 953374529.4 & 1210135920 & 1586333745 \\
\hline 10 & 1394308016 & 1677080584 & 2054749396 & 2574626772 & 3317285208 \\
\hline 11 & 1937283160 & 2312097672 & 2806472716 & 3476815011 & 4416841756 \\
\hline 12 & 1719752427 & 2062122789 & 2516919443 & 3138766641 & 4019509302 \\
\hline 13 & 944506803.7 & 1145417551 & 1417514780 & 1798578388 & 2354956160 \\
\hline 14 & 391150076.7 & 474085023.4 & 586602965.3 & 744748265.5 & 977257418.2 \\
\hline 15 & 661858656.9 & 795844411.4 & 975086970.6 & 1222576585 & 1578088353 \\
\hline 16 & 1549034100 & 1842368753 & 2227408027 & 2746663012 & 3470412397 \\
\hline 17 & 2231349792 & 2657722418 & 3217453718 & 3971693256 & 5020475115 \\
\hline 18 & 2040377920 & 2477311705 & 3068119651 & 3892521186 & 5087534069 \\
\hline 19 & 1075704199 & 1334990707 & 1699805390 & 2235530577 & 3066378137 \\
\hline 20 & 182912446.7 & 228615533.4 & 293854012.3 & 391576469.5 & 547542094.3 \\
\hline
\end{tabular}

So, the qualitative rule base based on the modal strain energy cloud reasoning of simply supported beam is formulated as: if $A_{r f}$, then $B_{r f}, r=1,2, \ldots, 20, f=1,2,3,4,5$.

The forward and backward cloud generator of qualitative rules can be regarded as qualitative concepts described with 3 digital characteristics of cloud model. For every rule in the rule base, we can construct the corresponding cloud generator and calculate by specific algorithm. In the cloud generator, many cloud droplets are produced with respective certainty degree as $\left(x_{b}^{f}, \mu_{f}\right)$, where $x_{b}^{f}$ is the quantitative output value of droplet in the $f$ th rule activated and $\mu_{f}$ is the certainty. According to weighting average method [25], the results is as $x_{b}=\sum_{f=1}^{m} x_{b}^{f} \mu_{f} / \sum_{f=1}^{m} \mu_{f}$.

In the Matlab software, the practical structure damage is simulated by reduction in element stiffness. Choose the first three modal vibration shape to calculate modal strain energy and measure 20 times under the 0.02 noise level. Choose the average value of the 20 times calculations results as the final identification result, so the identification effect is shown in Fig. 4 and Fig. 5 for the above two damage scenarios. 
Table 4. Entropy of damaged MSE in variable degrees of damage

\begin{tabular}{|c|c|c|c|c|c|}
\hline Element & Level 1 & Level 2 & Level 3 & Level 4 & Level 5 \\
\hline 1 & 132131618 & 144988877.6 & 160223634.4 & 228016033.2 & 262125195.9 \\
\hline 2 & 29644044.4 & 35158088.66 & 42603317.75 & 53109860.58 & 68872403.1 \\
\hline 3 & 13481788.27 & 16835321.44 & 21731945.46 & 29319905.59 & 42062542.65 \\
\hline 4 & 59369274.86 & 73464732.01 & 93551303.76 & 123567871.9 & 171175085 \\
\hline 5 & 97856807.53 & 118790809.7 & 147683793.7 & 189167507.4 & 241037368.7 \\
\hline 6 & 91812951.7 & 110679299.8 & 136501247 & 173246282.5 & 228108462.1 \\
\hline 7 & 53896753.44 & 65711619.64 & 82766366.02 & 106249977.3 & 143336318.2 \\
\hline 8 & 23949584.08 & 29506863.82 & 37418300.19 & 49268486.51 & 68241372.83 \\
\hline 9 & 35754315.95 & 44268550.67 & 56459364.07 & 74038927.91 & 104365011.4 \\
\hline 10 & 77155330.27 & 94373949.37 & 118471384.4 & 153693618.6 & 210125783.2 \\
\hline 11 & 105448005.7 & 127572033.9 & 158004886.6 & 201560305.1 & 233302516.2 \\
\hline 12 & 94542924.96 & 115117267.2 & 143684222.9 & 185022352.8 & 229838683.2 \\
\hline 13 & 53173078.95 & 65821047.3 & 83313099.08 & 110946708.3 & 154096722.8 \\
\hline 14 & 21994602.66 & 27233273.95 & 34754507.13 & 46142969.28 & 64640188.2 \\
\hline 15 & 36601045.97 & 44786579.66 & 56295733.73 & 74039988.2 & 99796314.43 \\
\hline 16 & 83698006.78 & 100796182.2 & 124178802.2 & 157433993.1 & 207090805.5 \\
\hline 17 & 120936701.2 & 145818017.6 & 179543596 & 234252526.4 & 299083635.1 \\
\hline 18 & 115150278.4 & 142581221.3 & 181513602.3 & 182662577.2 & 329510983.9 \\
\hline 19 & 63514491.27 & 81313612.54 & 108134955.1 & 151172195.3 & 224011491.4 \\
\hline 20 & 10956550.35 & 14191467.12 & 19183619.3 & 27473469.99 & 42685709.02 \\
\hline
\end{tabular}

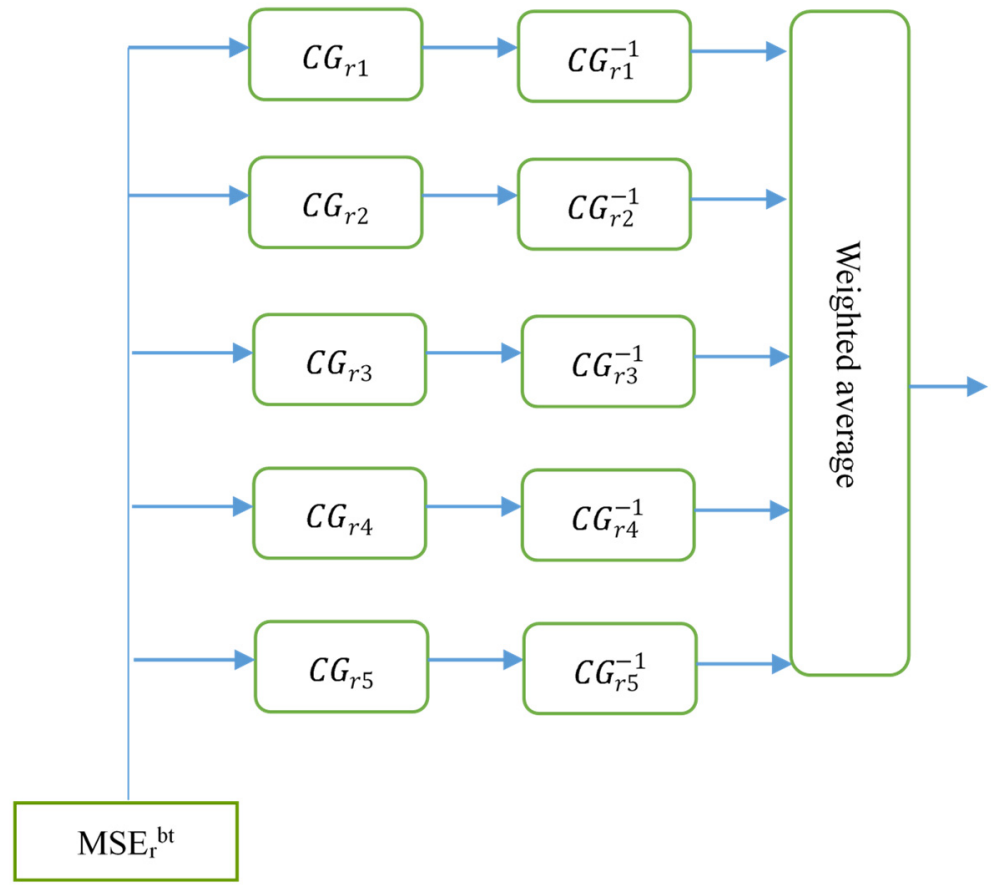

Fig. 3. Key flowchart of cloud reasoning based on MSE

For the cloud reasoning based on the acceleration inner product vector, this paper used a section of random white noise as the external excitation. To calculate the acceleration response of every measurement point, the Wilson- $\theta$ method is adopted. Furthermore, formulate the cross correlation function in the zero delay time to construct the acceleration inner product vector and the damage index $D_{i p v}$. The cloud reasoning method is similar as the cloud reasoning based on modal strain energy. And the identification results are listed as Fig. 6 and Fig. 7. 


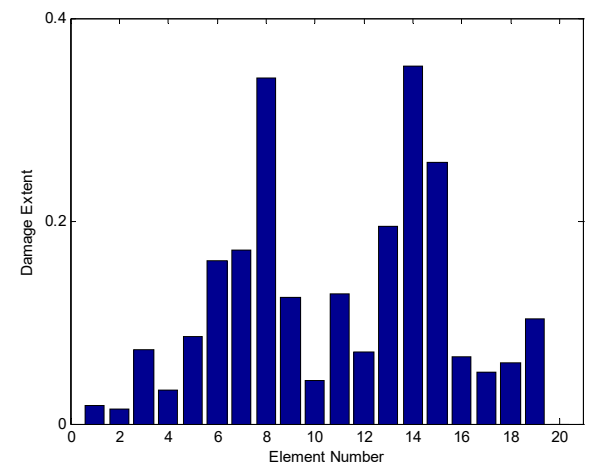

Fig. 4. Average identification result for scenario 1

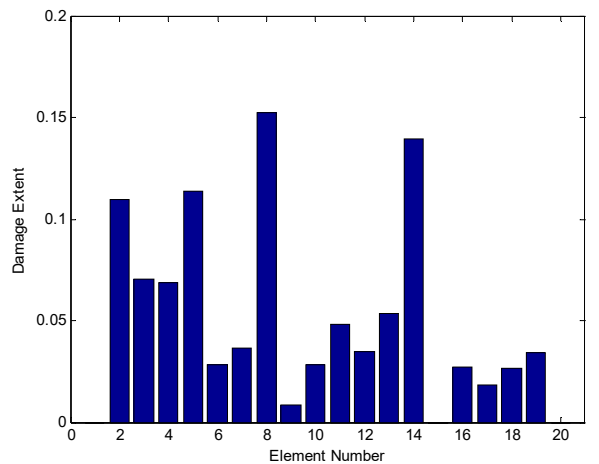

Fig. 6. Average identification result for scenario 1

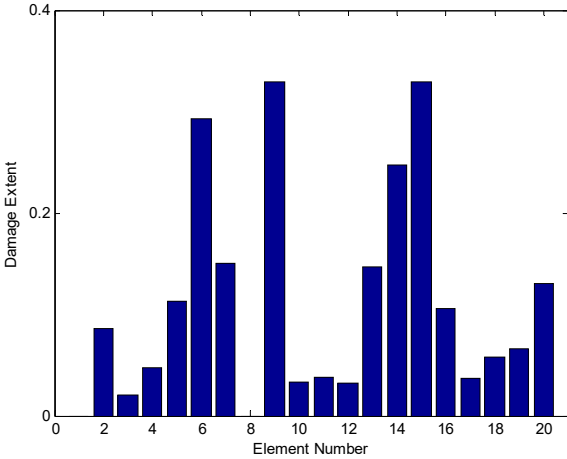

Fig. 5. Average identification for scenario 2

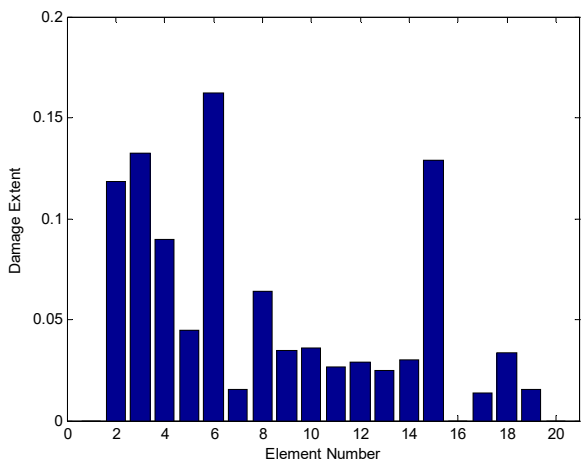

Fig. 7. Average identification result for scenario 2

Apply the improved D-S evidence theory into the above numerical example, the results are shown in Figs. 8-11.

As a supplement, the method based on modal strain energy dissipation rate is adopted to compare with the new method in based on cloud model and D-S theory. The results show that the new method has stronger anti-noise ability and higher accuracy.
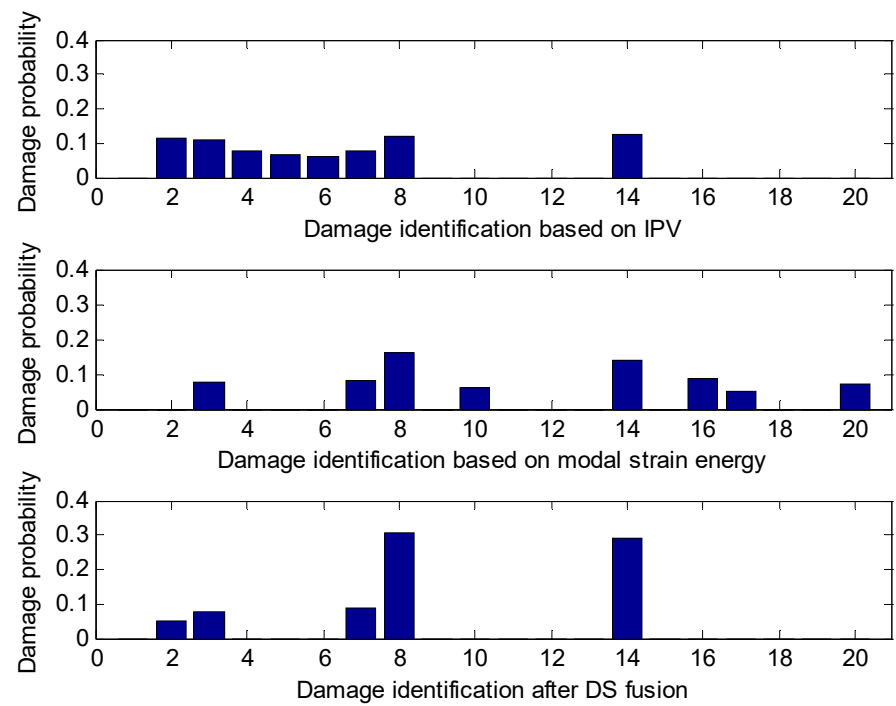

Fig. 8. Damage identification result with $2 \%$ noise for scenario 1 

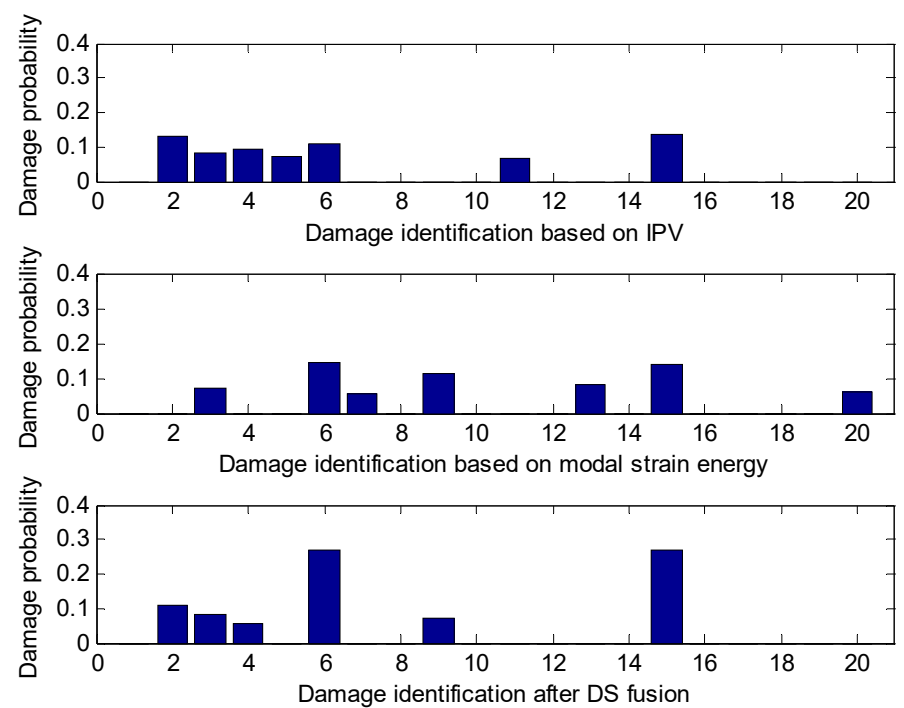

Fig. 9. Damage identification result with $2 \%$ noise for scenario 2

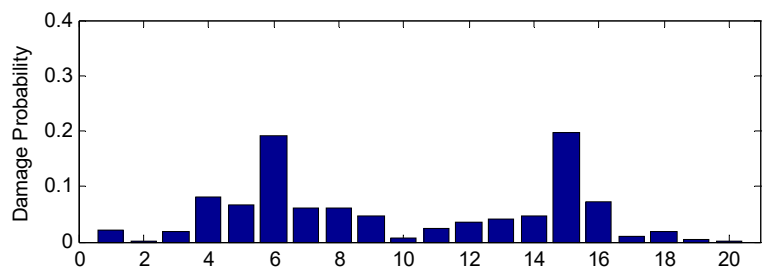

damage identification based on modal strain energy dissipation rate

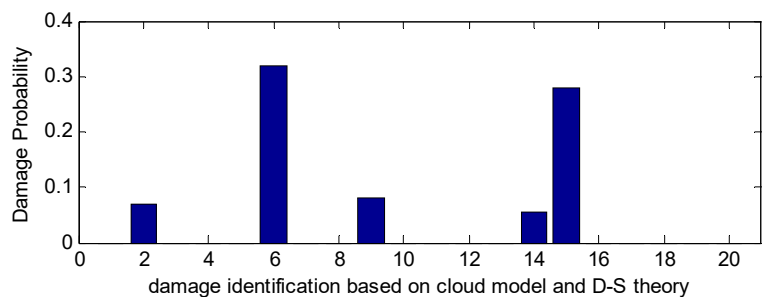

Fig. 10. Damage identification results without noise for scenario 2

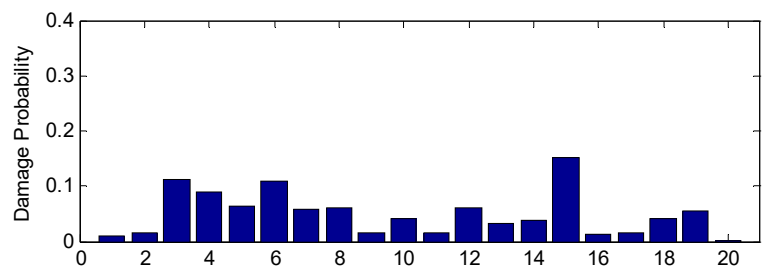

damage identification based on modal strain energy dissipation rate

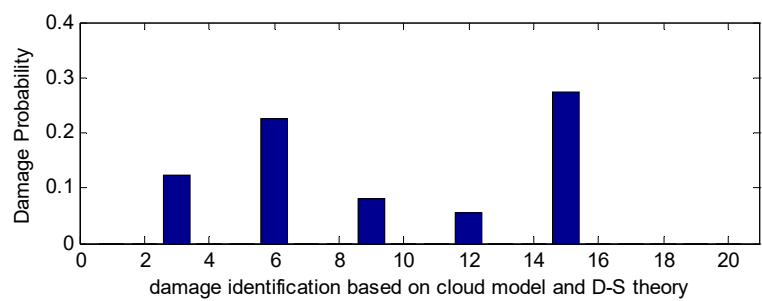

Fig. 11. Damage identification results with $2 \%$ noise for scenario 2 
Moreover, the original D-S theory was conducted to verify the effectiveness and necessity of the improved D-S theory. To save space, this paper only discussed the second damage scenario with $2 \%$ noise. And the results are listed in Table 5 and Fig. 12.

Table 5. The comparison of the original D-S and improved D-S in scenario 2 with $2 \%$ noise

\begin{tabular}{|c|c|c|}
\hline Element number & Original D-S & Improved D-S \\
\hline 1 & 0 & 0 \\
\hline 2 & 0 & 0 \\
\hline 3 & 0.079996 & 0.123399 \\
\hline 4 & 0 & 0 \\
\hline 5 & 0 & 0 \\
\hline 6 & 0.203831 & 0.218185 \\
\hline 7 & 0.053918 & 0 \\
\hline 8 & 0 & 0 \\
\hline 9 & 0.092054 & 0.075543 \\
\hline 10 & 0.066807 & 0 \\
\hline 11 & 0 & 0 \\
\hline 12 & 0.075728 & 0.05654 \\
\hline 13 & 0 & 0 \\
\hline 14 & 0 & 0 \\
\hline 15 & 0.249584 & 0.267519 \\
\hline 16 & 0 & 0 \\
\hline 17 & 0 & 0 \\
\hline 18 & 0 & 0 \\
\hline 19 & 0 & 0 \\
\hline 20 & 0 & 0 \\
\hline
\end{tabular}
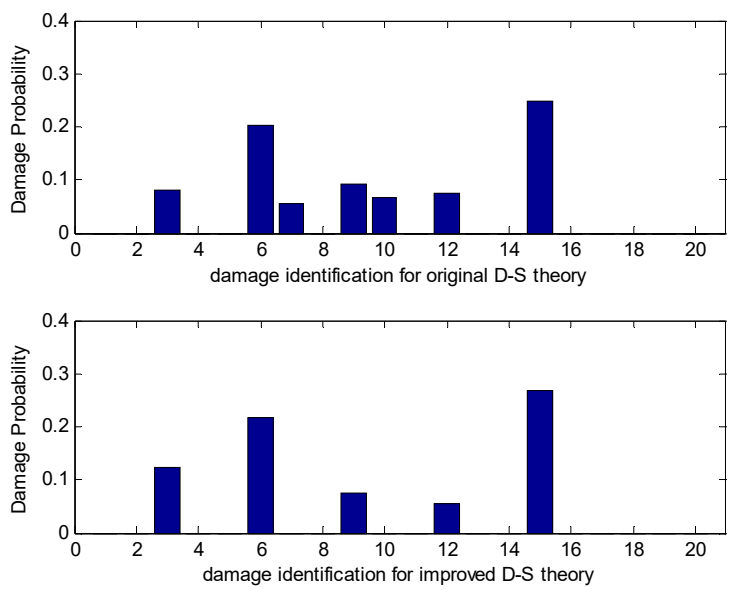

Fig. 12. The comparison of the original D-S and improved D-S in scenario 2 with $2 \%$ noise

From the comparison results between original D-S theory and improved D-S theory, we can draw the conclusion that the improved D-S theory is more accurate. In fact, with the numerical example becoming more complicated, the identification results of the improved D-S theory are more accurate than the original D-S theory.

In this paper, to assure a stable damage identification result of the structure under white noise, 20 times measurements are adopted, thus a matrix with order $20 \times 20$ is formed.

We calculated the average value, entropy value and excess entropy value of every element by the matrix to confirm the accuracy of this method. Meanwhile, the damage threshold was set at 0.005 so the element which is less than 0.005 can be neglected. Moreover, reliable index was formulated as $R_{i}=E_{n} / E_{x}$, where the $R_{i}$ is less, the result is more reliable. And if the $R_{i}$ is more 
than 1, it is shows that the results are divergent. Simultaneously, formulate the $R R_{i}=H_{e} / E_{n}$. The results of $R_{i}$ and $R R_{i}$ in two scenarios are listed in Table 6 and Table 7.

Table 6. Reliable index $R_{i}$ and $R R_{i}$ in scenario 1

\begin{tabular}{|c|c|c|}
\hline Damage element & 8 & 14 \\
\hline$R_{i}$ & & \\
\hline IPV & 1.2348 & 0.5143 \\
\hline Modal strain energy & 0.3438 & 0.6083 \\
\hline DS & 0.3404 & 0.4679 \\
\hline$R R_{i}$ & & \\
\hline IPV & 0.7995 & 0.6003 \\
\hline Modal strain energy & 2.6821 & 0.6449 \\
\hline DS & 1.5739 & 0.6855 \\
\hline
\end{tabular}

Table 7. Reliable index $R_{i}$ and $R R_{i}$ in scenario 2

\begin{tabular}{|c|c|c|}
\hline Damage element & 6 & 15 \\
\hline$R_{i}$ & & \\
\hline IPV & 0.9989 & 0.3194 \\
\hline Modal strain energy & 0.3822 & 0.8091 \\
\hline DS & 0.3324 & 0.2158 \\
\hline$R R_{i}$ & & \\
\hline IPV & 0.4699 & 1.0928 \\
\hline Modal strain energy & 0.5883 & 0.2818 \\
\hline DS & 1.3718 & 1.7759 \\
\hline
\end{tabular}

In the above texts, it can be easily seen that the results after D-S fusion is more accuracy and stable, which shows the practicality and effectiveness of improved D-S evidence theory.

\section{Conclusions}

Firstly, cloud models are created based on modal strain energy and acceleration Inner product vector, which can be calculated by structural vibration mode and acceleration separately. By apply the cloud models into a established simply supported beam model with white noise, the feasibility and anti-noise ability of cloud models are verified. To solve the defect of traditional D-S theory on fusing highly conflicting evidences, distance function is introduced and the accuracy of the improved D-S theory is proved. Meanwhile, the mean value, entropy and excess entropy of the results are utilized to analyze the reliability. The ultimate results show that the developed approach in this paper can be extensively applied in structural damage detection fields. Since the structural vibration mode and acceleration are easily measured in practical engineering, there is a bright application prospect of the new method.

\section{Acknowledgements}

This work was supported by the National Natural Science Foundation of China (Grant No. 51468058, 51578094), and the Fundamental Research Funds for the Central Universities (Grant No. 106112014CDJZR200011).

\section{References}

[1] Koh B. H., Dyke S. J. Structural health monitoring for flexible bridge structures using correlation and sensitivity of modal data. Computers and Structures, Vol. 85, Issues 3-4, 2007, p. 117-130.

[2] Shi Z. Y., Law S. S., Zhang L. M. Optimum sensor placement for structural damage detection. Journal of Engineering Mechanics, Vol. 126, Issue 11, 2000, p. 1173-1179. 
[3] Fan W., Qiao P. A 2-D continuous wavelet transform of mode shape data for damage detection of plate structures. International Journal of Solids and Structures, Vol. 46, Issues 25-26, 2009, p. 4379-4395.

[4] Seyedpoor S. M. A two stage method for structural damage detection using a modal strain energy based index and particle swarm optimization. International Journal of Non-Linear Mechanics, Vol. 47, Issue 1, 2012, p. 1-8.

[5] Guo H. Y., Li Z. L. Structural damage detection based on strain energy and evidence theory. Applied Mechanics and Materials, Vol. 48, Issues 49-2, 2011, p. 1122-1125.

[6] Jiang S. F., Zhang C. M., Zhang S. Two-stage structural damage detection using fuzzy neural networks nd data fusion techniques. Expert Systems with Applications, Vol. 38, Issue 1, 2011, p. 511-519.

[7] Dempster A. P. Upper and lower probabilities induced by a multi-valued mapping. Annals of Mathematical Statistics, Vol. 38, Issue 2, 1967, p. 325-339.

[8] Shafer G. A Mathematical Theory of Evidence. Princeton University Press, Princeton, NJ, 1976.

[9] Yang Z. C., Yu Z. F., Sun H. On the cross correlation function amplitude vector and its application to structural damage detection. Mechanical Systems and Signal Processing, Vol. 324, 2007, p. 2918-2932.

[10] Zhang M., Schmidt R. Sensitivity analysis of an auto-correlation-function-based damage index and its application in structural damage detection. Journal of Sound and Vibration, Vol. 333, Issue 26, 2014, p. 7352-7363.

[11] Fei Q., Li A., Han X. Simulation study on damage localization of a beam using evidence theory. Procedia Engineering, Vol. 1, Issue 1, 2009, p. 147-150.

[12] Li H., Bao Y., Ou J. Structural damage identification based on integration of information fusion and shannon entropy. Mechanical Systems and Signal Processing, Vol. 22, Issue 6, 2008, p. 1427-1440.

[13] Zhou Q., Zhou H., Zhou Q., et al. Structural damage detection based on posteriori probability support vector machine and Dempster-Shafer evidence theory. Applied Soft Computing, Vol. 36, 2015, p. 368-374.

[14] Li D. Y. Uncertainty in knowledge representation. Engineeringence, Vol. 2, Issue 10, 2000, p. 73-79.

[15] Li D. Y., Meng H., Shi X. Membership clouds and membership cloud generators. Journal of Computer Research and Development, Vol. 32, Issue 6, 1995, p. 1315-1325.

[16] Meng X., Zhang G., Kang J., et al. A new subjective trust model based on cloud model. IEEE International Conference on Networking, Sensing and Control, 2008, p. 1125-1130.

[17] Li D. Y., Liu C., Gan W. A new cognitive model: cloud model. International Journal of Intelligent Systems, Vol. 24, Issue 3, 2009, p. 357-375.

[18] Yuan S., De L. I., Xiao Y., et al. Reliability evaluation of electronic products based on cloud models. Acta Electronica Sinica, Vol. 28, Issue 12, 2000, p. 74-76.

[19] Liu C. Y., Feng M., Dai X. J., et al. New algorithm of backward cloud. Acta Simulata Systematica Sinica, Vol. 16, Issue 11, 2004, p. 2417-2420.

[20] Zhang W., Liu S., Sun B., et al. A cloud model-based method for the analysis of accelerated life test data. Microelectronics Reliability, Vol. 55, Issue 1, 2014, p. 123-128.

[21] Liu H., Qu W. L., Yuan R. Z. Structural damage detection method based on the theory of dissipation ratio of modal strain energy. Journal of Vibration and Shock, Vol. 23, Issue 2, 2004, p. 118-121, (in Chinese).

[22] Wang L., Yang Z. Effect of response type and excitation frequency range on the structural damage detection method using correlation functions of vibration responses. Journal of Sound and Vibration, Vol. 332, Issue 4, 2013, p. 645-653.

[23] Yang J. B., Wang Y. M., Xu D. L., et al. The evidential reasoning approach for MADA under both probabilistic and fuzzy uncertainties. European Journal of Operational Research, Vol. 171, Issue 1, 2006, p. 309-343.

[24] Jousselme A. L., Grenier D., Éloi Bossé A new distance between two bodies of evidence. Information Fusion, Vol. 2, Issue 2, 2001, p. 91-101.

[25] Jian G., Zhong L. I. Study on design and application of one-dimension cloud model mapping processor. Journal of System Simulation, Vol. 18, Issue 7, 2006, p. 1861-1865. 


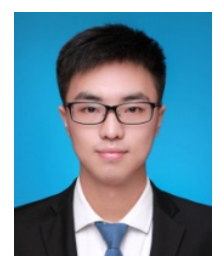

Liang Tian received Master degree in civil engineering from ChongQing University, ChongQing, China, in 2017. His current research interests include dynamics, damage identification and monitoring.

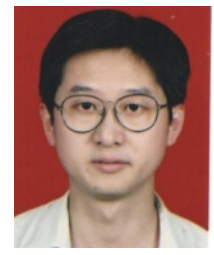

Huiyong Guo received Ph.D. degree in School of Aerospace from Xi'an Jiaotong University, Xi'an, China, in 2004. Now he works at School of Civil Engineering, Chongqing University, Chongqing, China. His current research interests include dynamics, damage identification and monitoring.

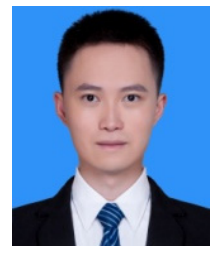

Xinyu Zhou is studying for a Master's degree in civil engineering from ChongQing University, ChongQing, China. His current research interests include dynamics, damage identification and monitoring.

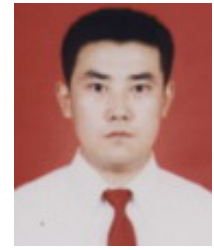

Yushan Wang received Master degree in civil engineering from Tianjin University, Tianjin, China, in 2009. Now he works at School of Civil Engineering, College of Water Conservancy and Architectural Engineering, Shihezi University, Shihezi, Xinjiang, China. His current research interests include structural seismic, damage identification and monitoring. 